\title{
C2 neurofibromas in neurofibromatosis type 1: genetic and imaging characteristics
}

\author{
Mueez Waqar, MRCS, MRes, ${ }^{1,2}$ Susan Huson, FRCP, ${ }^{2,3}$ D. Gareth Evans, FRCP, 2,3 \\ John Ealing, FRCP, ${ }^{2,4}$ Konstantina Karabatsou, FRCS, ${ }^{1,2} \mathrm{~K}$. Joshi George, FRCS, ${ }^{1,2}$ and \\ Calvin Soh, FRCR ${ }^{2,5}$
}

\begin{abstract}
Departments of ${ }^{1}$ Neurosurgery, ${ }^{4}$ Neurogenetics, and ${ }^{5}$ Neuroradiology, Salford Royal NHS Foundation Trust, Salford; ${ }^{3}$ Department of Neurogenetics, Manchester Centre for Genomic Medicine, St Mary's Hospital, Central Manchester University Hospitals NHS Foundation Trust, Manchester; and 'Manchester Academic Health Sciences Centre, Division of Evolution and Genomic Sciences, The University of Manchester, United Kingdom
\end{abstract}

OBJECTIVE C2 nerve root neurofibromas have been reported frequently in patients with neurofibromatosis type 1 (NF1), although their genetic and imaging characteristics are unexplored. The aim of this study was to characterize genetic and spinal imaging findings in a large cohort of NF1 patients with $\mathrm{C} 2$ neurofibromas.

METHODS The authors performed a review of national NF1 referrals between 2009 and 2016. Inclusion criteria were at least $1 \mathrm{C} 2$ root neurofibroma and cervical-spine or whole-spine MRI scans available for analysis. Blinded imaging review was performed by a neuroradiologist with an interest in NF1.

RESULTS Fifty-four patients with $106 \mathrm{C} 2$ neurofibromas were included. The median age was 32.5 years (range 15-61 years), and there were slightly more male patients (33 vs 21 female patients). Splice-site (30\%) and missense (20\%) variants were frequent. Spinal neurofibromas were distributed in all spine regions (65\%) or in the cervical spine alone (22\%). Most (93\%) C2 neurofibromas were visible on MRI scans of the head. Intradural invasion and cord compression in the cervical spine included the $\mathrm{C} 2$ level in $95 \%$ and $80 \%$ of patients, respectively. Compared with all other cervical spine neurofibromas in these patients, C2 neurofibromas had higher rates of intraspinal extension (75\% vs 32\%; OR 6.20, 95\% Cl 3.85-9.97; $p<0.001$ ), intradural invasion (53\% vs 26\%; OR 3.20, 95\% Cl 2.08-4.92; $p<0.001$ ), and cord compression (25\% vs $13 \%$; OR 2.26, 95\% Cl 1.35-3.79; $p=0.002)$. However, C2 neurofibromas had lower rates of extraforaminal growth beyond the transverse process ( $12 \%$ vs $62 \%$; OR 0.09, 95\% Cl 0.05-0.16; $p<0.001$ ).

CONCLUSIONS C2 neurofibromas are associated with an aggressive intraspinal phenotype, limited growth outside the spinal canal, and an uncommon genetic profile. These observations require future study.

https://thejns.org/doi/abs/10.3171/2018.7.SPINE171340

KEYWORDS neurofibromatosis type 1; Von Recklinghausen's disease; spinal neurofibromas; spinal neurofibromatosis; oncology

$\mathrm{N}$ EUROFIBROMATOSIS type 1 (NF1) is a common autosomal-dominant condition with a reported incidence of around 1 in $2500 .{ }^{7} \mathrm{NF} 1$ patients can have a variety of spinal pathologies, including structural deformities of the spine, dural ectasia, developmental bony abnormalities, cord meningocele, and segmental and plexiform neurofibromas. ${ }^{18}$

Segmental spinal neurofibromas have a reported prevalence of up to $40 \%$ in NF1 patients..$^{23}$ Reported rates vary by institution due to differences in criteria for spinal imaging. Neurofibromas affecting the $\mathrm{C} 2$ nerve root in par- ticular have been described in patients with and without NF1., ${ }^{1,511,14,22}$ Although existing studies are small, C2 neurofibromas appear to have high rates of intradural invasion and cord compression. ${ }^{14}$ The reason for this observation is not entirely clear, although it may reflect vulnerability of the $\mathrm{C} 2$ nerve root to low-grade trauma along its course over the superior aspect of the C2 lamina. ${ }^{14}$

Cervical spine neurofibromas have a high rate of intraspinal growth in general; ${ }^{2,25}$ a comparison between $\mathrm{C} 2$ neurofibromas and other segmental cervical spine neurofibromas has not yet been presented. The aim of this study 
was to characterize genetics and spinal imaging findings in a large cohort of NF1 patients with C2 neurofibromas. We also sought to systematically compare $\mathrm{C} 2$ neurofibromas to other cervical spine neurofibromas in these patients.

\section{Methods}

\section{Patient Identification and Data Collection}

Our study was approved by the institutional review board. Salford Royal NHS Foundation Trust and Central Manchester University Hospitals NHS Foundation Trust together represent a national NF1 referral center in the UK as one of 2 nationally funded, highly specialized services. A prospective database of NF1 referrals (1109 patients) was queried for C2 neurofibromas between 2009 and 2016. Adult patients meeting the National Institutes of Health criteria for NF1 with at least $1 \mathrm{C} 2$ nerve root neurofibroma demonstrable on cervical-spine or whole-spine MRI were included. Patient demographic, presentation, and genetic data were obtained through electronic records.

\section{Institutional Practice}

In general, spinal imaging is performed when spinal pathology is visualized on other imaging sequences (e.g., head MRI) or when clinically indicated (e.g., myelopathy, neck pain or neck lump). NFI gene testing is not always performed as per guidance. ${ }^{8}$

\section{Imaging Review}

Imaging review was performed by a single neuroradiologist with a specialist interest in NF1 (C.S.), who was blinded to clinical data. T1- and T2-weighted cervicalspine and whole-spine MRI sequences with or without contrast were reviewed. The following imaging characteristics were recorded for $\mathrm{C} 2$ neurofibromas: tumor laterality (unilateral or bilateral), intradural extension (present or absent-a hallmark of dural penetration were noncontiguous segments of dura visible on either side of the tumor), extent of spinal canal occlusion where applicable $(0,0 \%-25 \%$, $25 \%-50 \%, 50 \%-75 \%$, and $75 \%-100 \%$ ), cord compression (present or absent), and extraforaminal extension (up to the transverse process, beyond the transverse process). These characteristics were also recorded for other cervical spine tumors. Thoracic and lumbar spine neurofibromas were characterized according to the presence or absence of single or multilevel intradural disease or cord compression. Head MRI scans of included patients were also reviewed to determine the visibility of $\mathrm{C} 2$ neurofibromas in any of the sequences obtained in different orthogonal planes.

\section{Outcomes and Statistics}

Statistical analysis was performed using IBM SPSS (version 20, IBM Corp.). Descriptive statistics were used to describe tumor characteristics. Group comparisons were performed using odds ratios and $95 \%$ confidence intervals. Progression was defined as an increase in tumor dimensions on serial imaging. Progression-free survival (PFS) was the maximum time period in months between serial images without evidence of progression. All C2 neurofibromas were evaluated for progression.

\section{Results \\ Patient Demographics}

A total of 126 NF1 patients with spinal neurofibromas were referred to our center during the study period. Fiftyfour NF1 patients with at least 1 C2 neurofibroma were included in this study, representing $43 \%$ of patients with spinal neurofibromas. The median age was 32.5 (range 15-61) years. There was a slight male preponderance (33 vs 21 female patients).

\section{Neurological and Genetic Characteristics}

The majority of patients $(32 / 54,59 \%)$ were asymptomatic, and the $\mathrm{C} 2$ neurofibroma was discovered incidentally. Clinical features prompting radiological investigations included myelopathy $(17 / 54,31 \%)$, neck lump $(2,4 \%)$ and significant neck pain $(2,4 \%)$.

Gene analysis was performed in 30 patients. Exon variants $(16,53 \%)$ were more common than intronic variants $(7,23 \%)$. Splice-site variants $(9,30 \%)$ were less common than non-splice-site variants $(15,50 \%)$. Missense $(6,20 \%)$ and nonsense/frame shift $(7,23 \%)$ variants occurred in a similar proportion of patients. Four patients $(13 \%)$ had whole gene deletions: 2 had promotor region (5'-untranslated region) pathogenic variants and $2(7 \%)$ had no identifiable variant.

\section{Extent of Spinal Disease}

Forty-nine patients underwent whole-spine MRI. Concurrent spinal disease included scoliosis $(30 / 49,61 \%)$, dural ectasia $(17 / 49,35 \%)$, developmental vertebral anomalies $(4 / 49,8 \%)$, and a syrinx $(2 / 49,4 \%)$. Most patients $(32 / 49,65 \%)$ had segmental neurofibromas in all 3 spine regions (cervical, thoracic, and lumbosacral). The second most common subgroup $(11 / 49,22 \%)$ had isolated cervical spine segmental neurofibromas. Three patients $(6 \%)$ had isolated neurofibromas at the $\mathrm{C} 2$ level without segmental neurofibromas elsewhere in the spine.

Intradural invasion and cord compression were highest in the cervical spine and lowest in the thoracic spine (Fig. 1).

Single-level and multilevel intradural invasion in the cervical spine included the C2 level in 14 of $15(93 \%)$ and 24 of $25(96 \%)$ patients, respectively. Single-level and multilevel cord compression in the cervical spine included the C2 level in 6 of $11(55 \%)$ and 14 of 14 (100\%) patients, respectively. Overall, intradural invasion and cord compression in the cervical spine included the $\mathrm{C} 2$ level in $95 \%$ and $80 \%$ of patients, respectively.

\section{C2 Neurofibroma Characteristics}

There was an extremely high rate of bilateral involvement of the $106 \mathrm{C} 2$ neurofibromas in the 54 patients, with 52 patients having bilateral tumors and 2 having unilateral tumors. Forty-three patients had MRI head studies available for analysis, and $\mathrm{C} 2$ neurofibromas were visible on 40 of 43 scans $(93 \%)$.

Imaging characteristics are summarized in Table 1 and presented in Fig. 2. C2 neurofibromas had a high rate of intraspinal extension $(79 / 106,75 \%)$, intradural invasion (56/106, 53\%), and cord compression or impingement 
A

No intradural invasion $\quad$ Single-level intradural invasion Multi-level intradural invasion

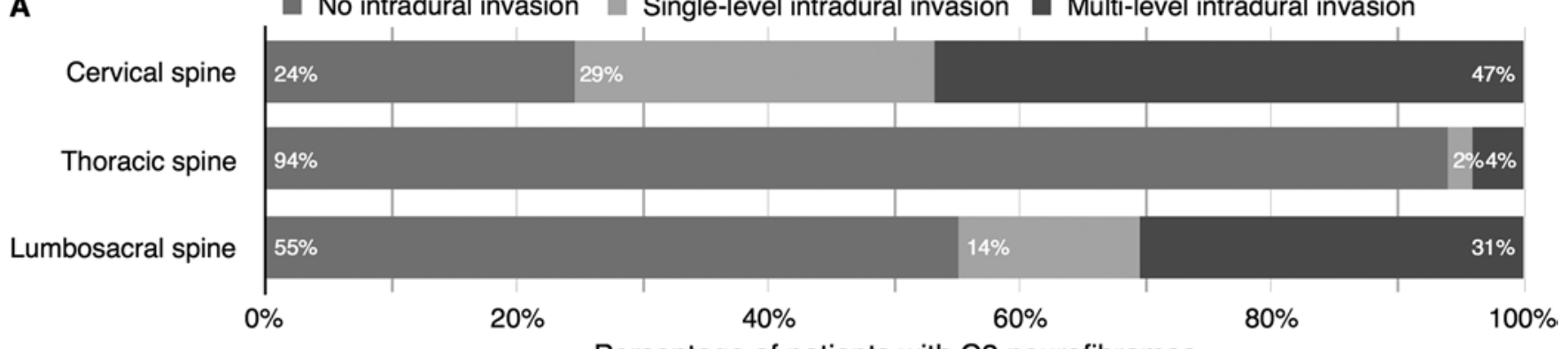

Percentage of patients with C2 neurofibromas

B

No cord compression $\quad$ Single-level cord compression $\quad$ Multi-level cord compression

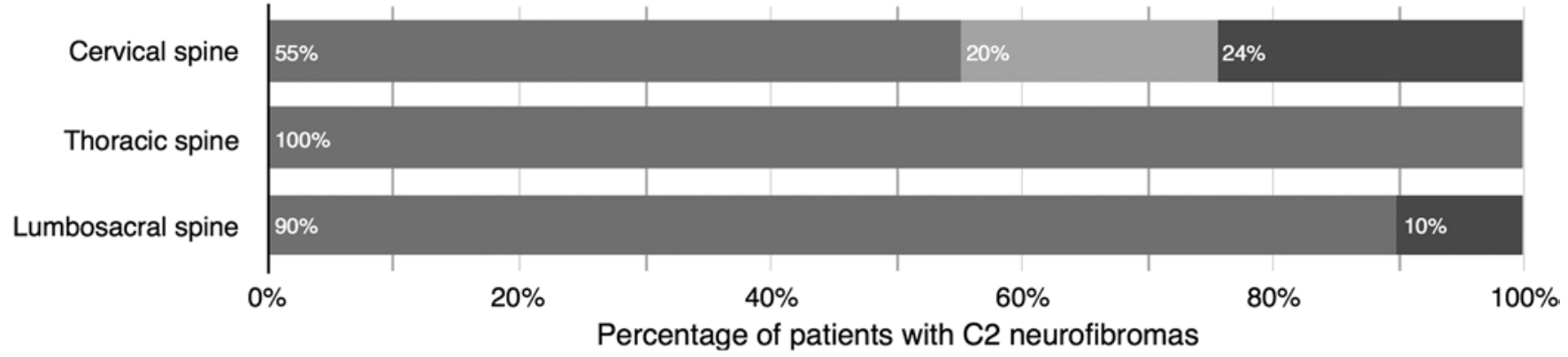

FIG. 1. Spine segmental neurofibroma patterns in patients with C2 neurofibromas. A: Intradural invasion patterns. These were similar between the cervical and lumbosacral spine. Intradural invasion was rare in the thoracic spine. B: Cord compression patterns. In general, radiological cord compression was rare outside of the cervical spine.

(47/106, 44\%). Extraforaminal size was more limited and usually extended to the transverse process $(93 / 106,88 \%)$.

\section{Cervical Segmental Neurofibroma Comparisons}

Upper (C1-4) Versus Lower (C5-8) Cervical Segmental Neurofibromas

Upper (C1-4) cervical segmental neurofibromas had higher rates of intraspinal extension $(52 \%$ vs $28 \%$; OR $2.85,95 \%$ CI 2.03-3.98; $\mathrm{p}<0.001$ ), intradural invasion (38\% vs 23\%; OR 2.05, 95\% CI 1.45-2.92; p < 0.001), and cord compression (18\% vs $12 \%$; OR $1.56,95 \%$ CI $0.99-2.46 ; \mathrm{p}=0.05$ ) (Table 2). However, the upper-level segments had significantly lower rates of extraforaminal growth beyond the transverse process (20\% vs $84 \%$; OR $0.05,95 \% 0.03-0.07 ; \mathrm{p}<0.001)$.

\section{C2 Neurofibromas Versus All Other Cervical Segmental} Neurofibromas

C2 neurofibromas had higher rates of intraspinal extension (75\% vs 32\%; OR $6.20,95 \%$ CI 3.85-9.97; p < 0.001 ), intradural invasion (53\% vs $26 \%$; OR $3.20,95 \%$ CI $2.08-4.92 ; \mathrm{p}<0.001)$, and cord compression (25\% vs 13\%; OR 2.26, 95\% CI 1.35-3.79; $p=0.002$ ). However, they had lower rates of extraforaminal growth beyond the transverse process than all other cervical neurofibromas (12\% vs 62\%; OR 0.09, 95\% CI 0.05-0.16; $\mathrm{p}<0.001$ ).

C2 Neurofibromas Versus Other Upper (C1, C3, and C4) Cervical Segmental Neurofibromas

$\mathrm{C} 2$ neurofibromas had higher rates of intraspinal extension (75\% vs 40\%; OR 4.49, 95\% CI 2.65-7.60; p <
TABLE 1. Imaging characteristics of $\mathrm{C} 2$ nerve root neurofibromas

\begin{tabular}{lc}
\hline & No. $(\%)$ \\
\hline Total no. & 106 \\
\hline Tumor laterality* & $52(96)$ \\
\hline Bilateral & $2(4)$ \\
\hline Unilateral & \\
\hline Larger component of bilateral tumors* & $16(31)$ \\
\hline Lt & $19(37)$ \\
\hline Rt & $17(33)$ \\
\hline Equal sized & \\
\hline Extraforaminal size & $93(88)$ \\
\hline Btwn nerve root \& transverse process & $13(12)$ \\
\hline Extends beyond transverse process & $79(75)$ \\
\hline Intraspinal extension & $47 / 79(59)$ \\
\hline Total & $27 / 79(34)$ \\
\hline $0-25 \%$ & $4 / 79(5)$ \\
\hline $25-50 \%$ & $1 / 79(1)$ \\
\hline $50-75 \%$ & $56(53)$ \\
\hline $75-100 \%$ & $21(20)$ \\
\hline Intradural invasion & $26(25)$ \\
\hline Cord change & \\
\hline Impingement & \\
\hline Compression & \\
\hline
\end{tabular}

Values are presented as the number of $\mathrm{C} 2$ neurofibromas (\%) unless stated otherwise.

${ }^{*}$ Values are number of patients (\%). 
Waqar et al.
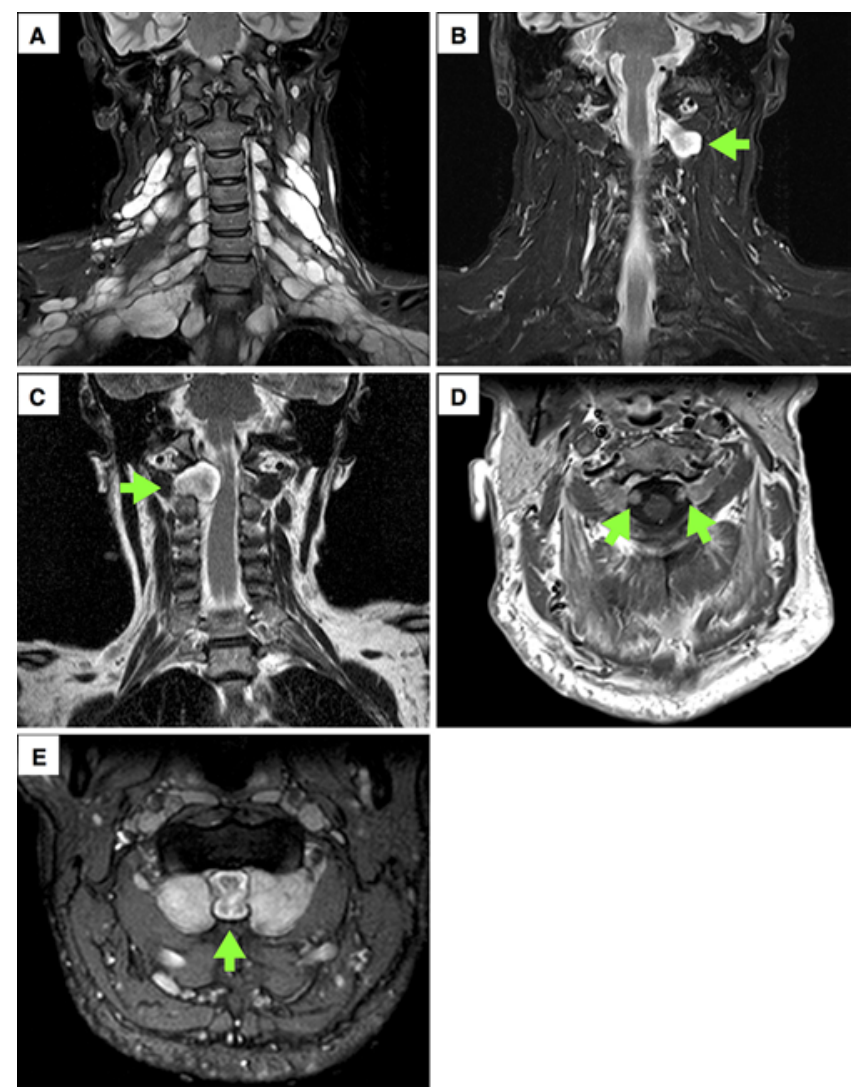

FIG. 2. Features in patients with $C 2$ neurofibromas as seen on coronal $(A-C)$ and axial (D and E) images. A: Multilevel segmental spinal neurofibromas were common, as in this 39-year-old man with extensive segmental neurofibromas throughout the whole spine, including plexiform neurofibromatous involvement of the brachial plexi and lumbar and sacral plexi. B: Isolated neurofibromas were rare, as in this 61-year-man male with an isolated, unilateral left-sided C2 neurofibroma (arrow), without neurofibromas elsewhere in the spine. C: Some neurofibromas had intraspinal growth without intradural invasion, as in this 52-year-old woman with an extensive right-sided $\mathrm{C} 2$ neurofibroma causing cord compression (arrow). D: Intradural invasion was present in a significant proportion, as in this 45-year-old man with bilateral C2 neurofibromas with intradural invasion (1\%-25\%; arrow). E: Radiological cord compression was commonly associated with $\mathrm{C} 2$ neurofibromas, as in this 19-year-old woman with progressive myelopathy (arrow) due to bilateral $\mathrm{C} 2$ neurofibromas. Figure is available in color online only.

0.001 ), intradural invasion (53\% vs $30 \%$; OR $2.58,95 \%$ CI 1.57-3.76; $\mathrm{p}<0.001)$, and cord compression (25\% vs 14\%; OR 2.08, 95\% CI 1.13-3.83; $\mathrm{p}=0.02$ ). Although C2 neurofibromas had lower rates of extraforaminal growth, differences were not statistically significant ( $12 \%$ vs $24 \%$; OR $0.44,95 \%$ CI $0.23-0.88 ; \mathrm{p}=0.55$ ).

\section{Outcome}

Follow-up imaging was available in 42 patients. The median radiological follow-up period was 34 months (range 3-95 months). Radiological progression occurred in 5 of $42(12 \%)$ patients with C2 neurofibromas during the follow-up period. The median PFS was not reached. The 2- and 5-year PFS rates were $97 \%$ and $84 \%$, respectively.

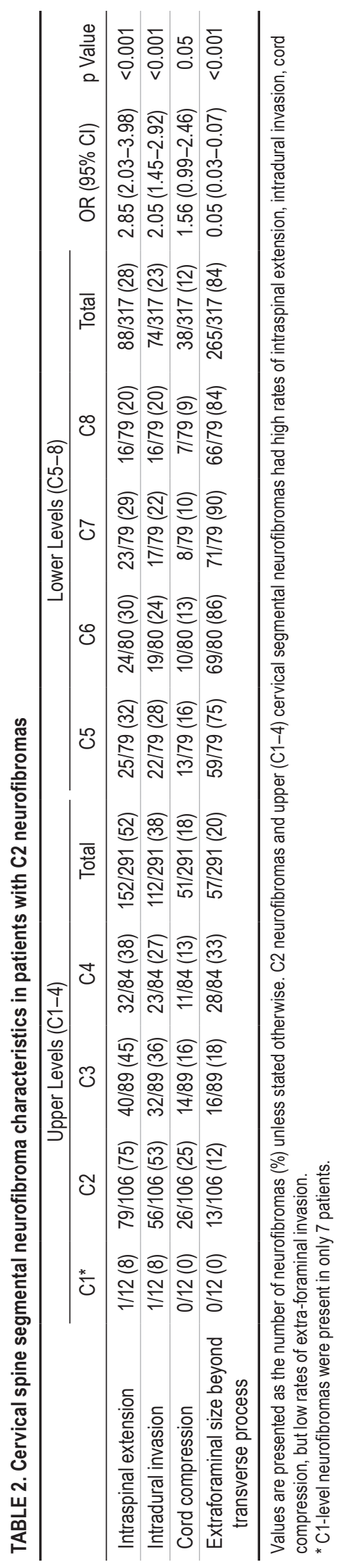

J Neurosurg Spine Volume $30 \cdot$ January 2019 


\section{Discussion}

In this study, we demonstrated the aggressive intraspinal nature of C2 neurofibromas in a large cohort of NF1 patients. Genetic analysis revealed a high frequency of splice-site and missense variants. Most patients had concurrent spinal pathology, such as deformity and dural ectasia. Spinal segmental neurofibromas were distributed in all spine regions in two-thirds of patients, or restricted to the cervical spine in around one-quarter. Intradural invasion and cord compression were most common in the cervical spine and usually involved the $\mathrm{C} 2$ level. Upper (C1-4) cervical neurofibromas had a more aggressive intraspinal growth pattern, whereas lower $(\mathrm{C} 5-8)$ cervical neurofibromas tended to grow outside the spinal canal. Compared with other cervical spine neurofibromas, C2 neurofibromas had significantly higher rates of intraspinal extension, intradural invasion, and radiological cord compression.

\section{Genetic Considerations}

The NF1 gene can have several pathogenic variant types, as we have reported. ${ }^{6}$ In a typical cohort of patients with classic NF1, these variants include introns in $11 \%$, splice-site in $22 \%$, missense in $9 \%$, nonsense in $21 \%$, and whole gene deletion in $6 \%{ }^{6}$ In the present study, a higher frequency of splice-site (30\%) and missense (20\%) variants was observed. This corresponds to studies including NF1 patients with a high burden of spinal disease, who may only have limited classic cutaneous findings. ${ }^{4,21} \mathrm{Al}-$ though the exact reasons for this observation are unclear, one possibility is as follows. The mRNA transcribed from regions containing splice-site and missense variants usually escapes degradation, and the resulting protein could therefore have partial function. However, a milder NF1 pathogenic variant does not explain potentially aggressive spinal disease, which may instead result from the interaction of the NFl gene and other factors such as modifier genes. ${ }^{21}$ Further studies are required to better understand genotype-phenotype correlations in such patients.

\section{Spinal Disease}

Spinal disease in patients with NF1 is common, and the reported prevalence varies depending on the institutional imaging protocol. Recently, Nguyen et al. described the prevalence of spinal pathology in NF1 patients enrolled in a natural history study. ${ }^{18}$ Although their patient cohort had a more severe NF1 phenotype, $90 \%$ had at least one abnormal finding on spinal MRI. Scoliotic deformity was the most common finding, present in half of their cohort. Scoliosis has a high prevalence in NF1, reported in 2\%-69\% of patients. ${ }^{24}$ It has been shown to correlate with spinal tumor burden, and our results would support this finding. ${ }^{18}$

\section{Prevalence of Neurofibromas}

Neurofibromas have a high prevalence at the $\mathrm{C} 2$ level in patients with and without NF1. ${ }^{1,5,11,14,22}$ In studies including predominantly non-NF1 patients, C2 neurofibromas constitute $5 \%-25 \%$ of peripheral nerve sheath tumors in the spine. ${ }^{10,11,16}$ The rate is higher in patients with NF1. In one study of NF1 patients undergoing surgery for symptom- atic cervical spine neurofibromas, 9 of 22 (41\%) patients had neurofibromas at the $\mathrm{C} 2$ level. ${ }^{22}$ In fact, it was the most common level affected. Another small study reported that the C2 level was affected in 12 of $13(92 \%)$ patients presenting with cervical spine cord compression. ${ }^{14}$ In our larger series, C2 neurofibromas were present in about $40 \%$ of NF1 patients with spinal neurofibromas.

There was a low prevalence of $\mathrm{C} 1$ neurofibromas in the present study, which is similar to that in earlier reports. George and Lot described a series of $50 \mathrm{C} 1$ and $\mathrm{C} 2$ neurofibromas and schwannomas, of which only $6(12 \%)$ occurred at the $\mathrm{C} 1$ level. ${ }^{10}$ Abe et al. also reported $\mathrm{C} 1$ neurofibromas in 3 of 24 (13\%) patients who underwent surgery for cervical neurofibromas. ${ }^{1}$

\section{Phenotype of C2 Neurofibromas}

Our results provide evidence as to the aggressive intraspinal nature of upper cervical region neurofibromas in patients with NF1, particularly C2 neurofibromas. Myelopathy is commonly associated with $\mathrm{C} 2$ neurofibromas. ${ }^{14,17}$ We found that the majority of these tumors tend to occur bilaterally, with high rates of intradural invasion. The rate of intradural invasion in our study was higher than that reported in other studies. Jinnai and Koyama described the characteristics of 176 surgically treated neurofibromas in 149 patients, most (137/149) of whom did not have NF1..$^{13}$ Of 28 surgically treated $\mathrm{C} 1-2$ neurofibromas, only $7(25 \%)$ had evidence of intradural invasion. Goel et al. described 66 surgically managed patients with $\mathrm{C} 2$ neurofibromas, of whom 9 had NF1 (15\%). ${ }^{11}$ Intradural invasion was present in 12 patients (18\%). Another surgical series included $8 \mathrm{C} 2$ neurofibromas, although only one had evidence of intradural invasion. ${ }^{1}$ El-Sissy and Mahmoud reported a series of 11 patients who underwent debulking of $\mathrm{C} 2$ neurofibromas and schwannomas, all of whom were described to have intradural invasion. ${ }^{5}$ As these studies have reported findings in mostly non-NF1 patients, it is interesting to note the more aggressive findings we have found in a group of NF1 patients. This is corroborated by studies finding a high frequency of these tumors in symptomatic NF1 patients..$^{14,22}$

The aggressive phenotype of $\mathrm{C} 2$ neurofibromas could be explained by the anatomy of the $\mathrm{C} 2$ nerve root. The $\mathrm{C} 2$ ganglion is the largest in the spinal cord and is sandwiched between the overlying posterior arch of $\mathrm{C} 1$ and underlying lateral mass of $\mathrm{C} 2 .^{11,12}$ The $\mathrm{C} 1-2$ level is also unique in having the most rotational movement in the spine. These factors predispose the nerve root to repeated trauma from even minor cervical spine and head movement. Whether trauma actually induces neurofibroma formation is controversial, and current evidence centers around dermal neurofibromas. There are at least 3 mechanisms relating trauma to neurofibroma formation. 1) NF1 is a regulator of fibroblast function and murine knockout models have displayed aberrant fibroblast proliferation, which could occur in response to trauma. ${ }^{3}$ 2) Nerve injury induces Schwann cell proliferation and the likelihood of a "second-hit" to the NF1 gene. $\left.{ }^{19} 3\right)$ Injury appears to create a protumorigenic environment through special signaling pathways, which could also increase the likelihood of tumor formation. ${ }^{19}$ However, there is also evidence to the contrary. NF1 
patients undergoing surgery do not have an increased incidence of neurofibroma formation at the surgical site, as would be expected from this hypothesis..$^{9,20}$

Trauma-induced neurofibroma growth does not easily explain the predilection for intraspinal versus extraforaminal growth of upper cervical neurofibromas. The spinal canal dimensions actually decrease sequentially from the C1-2 level to C7. ${ }^{15}$ First, this means that upper cervical nerve root neurofibromas have to enlarge to a much greater size in comparison to lower cervical neurofibromas in order to cause cord compression. Second, if trauma promotes the growth of these tumors, it would suggest that most of the nerve irritation occurs intraspinally at upper versus lower cervical levels. Unfortunately, there are no data to support this hypothesis. Our findings are also contrary to those of Jinnai and Koyama, who found that the frequency of extraforaminal growth decreased down the cervical spine. The authors found a much higher rate of extraforaminal growth in C1-2 tumors versus C3-8 neurofibromas. ${ }^{13}$

\section{Tumor Progression}

Radiological neurofibroma progression at the C2 level occurred in $12 \%$ of patients with follow-up imaging. Follow-up imaging was not available in all patients, as imaging is generally only repeated if there is a change in symptoms or signs, which would also prompt re-referral to our service. Other NF1 series have analyzed progression at multiple spinal levels and thus generally report a higher rate of tumor progression than that in the present study. For example, in the study by Nguyen et al., 9 of 26 children with NF1 with spinal neurofibromas had radiological progression of paraspinal neurofibromas. ${ }^{18}$

\section{Identification on MRI of the Head}

The majority $(93 \%)$ of C2 neurofibromas were clearly identified in at least one of the 3 orthogonal planes. The lesion was most conspicuous if axial imaging commenced at the level of the $\mathrm{C} 2$ body. Coronal and axial images best demonstrate the intradural components. In 3 patients, the C2 neurofibromas were not visualized on MRI head scans alone. This was either because axial imaging commenced at the level of the foramen magnum, excluding the $\mathrm{C} 2$ level from the imaging field, or the lesions were too small to permit confident identification. The significance of these findings is shown by the fact that, in some patients, spinal imaging was performed after identification of C2 lesions on brain imaging.

\section{Limitations}

The current study represents a cross-sectional analysis of mainly cervical spine neurofibromas in patients with $\mathrm{C} 2$ neurofibromas. Patients without C2 neurofibromas were not included in this study, such that the true aggressiveness in general of neurofibromas arising at spinal levels other than C2 cannot be ascertained using the current methodology. Follow-up imaging was not analyzed to determine tumor progression for levels other than $\mathrm{C} 2$. We also did not analyze other spinal levels to the same extent as the cervical spine, as this was beyond the scope of this study.
Neurofibromas reported in this study were identified on the basis of imaging appearance rather than histology, although a specialist neuroradiologist with interest in NF1 reviewed all scans.

\section{Conclusions}

In this cross-sectional study from a national NF1 center, we demonstrated the aggressive intraspinal nature of C2 neurofibromas in a large cohort of NF1 patients. These tumors are usually visible on MRI scans of the head, which should prompt further spinal imaging due to the high frequency of concurrent spinal pathology. In patients with C2 neurofibromas, intradural invasion and cord compression associated with neurofibromas in the cervical spine usually involve the $\mathrm{C} 2$ level. Furthermore, although upper (C1-4) cervical neurofibromas display an aggressive intraspinal growth pattern in general, the $\mathrm{C} 2$ level is the most aggressive in this patient group. Future studies are required to better understand these observations.

\section{Acknowledgments}

We acknowledge Miss Lauren Lewis, Central Manchester NHS Foundation Trust, for assistance with identifying the cohort of patients.

\section{References}

1. Abe J, Takami T, Naito K, Yamagata T, Arima H, Ohata K: Surgical management of solitary nerve sheath tumors of the cervical spine: a retrospective case analysis based on tumor location and extension. Neurol Med Chir (Tokyo) 54:924929, 2014

2. Ars E, Kruyer H, Gaona A, Casquero P, Rosell J, Volpini V, et al: A clinical variant of neurofibromatosis type 1: familial spinal neurofibromatosis with a frameshift mutation in the NF1 gene. Am J Hum Genet 62:834-841, 1998

3. Atit RP, Crowe MJ, Greenhalgh DG, Wenstrup RJ, Ratner $\mathrm{N}$ : The Nf1 tumor suppressor regulates mouse skin wound healing, fibroblast proliferation, and collagen deposited by fibroblasts. J Invest Dermatol 112:835-842, 1999

4. Burkitt Wright EM, Sach E, Sharif S, Quarrell O, Carroll T, Whitehouse RW, et al: Can the diagnosis of NF1 be excluded clinically? A lack of pigmentary findings in families with spinal neurofibromatosis demonstrates a limitation of clinical diagnosis. J Med Genet 50:606-613, 2013

5. El-Sissy MH, Mahmoud M: C2 root nerve sheath tumors management. Acta Neurochir (Wien) 155:779-784, 2013

6. Evans DG, Bowers N, Burkitt-Wright E, Miles E, Garg S, Scott-Kitching V, et al: Comprehensive RNA analysis of the NF1 gene in classically affected NF1 affected individuals meeting NIH criteria has high sensitivity and mutation negative testing is reassuring in isolated cases with pigmentary features only. EBioMedicine 7:212-220, 2016

7. Evans DG, Howard E, Giblin C, Clancy T, Spencer H, Huson $\mathrm{SM}$, et al: Birth incidence and prevalence of tumor-prone syndromes: estimates from a UK family genetic register service. Am J Med Genet A 152A:327-332, 2010

8. Ferner RE, Huson SM, Thomas N, Moss C, Willshaw H, Evans DG, et al: Guidelines for the diagnosis and management of individuals with neurofibromatosis 1. J Med Genet 44:81-88, 2007

9. Friedrich RE, Hagel C, Brehme Z, Kluwe L, Mautner VF: Ki-67 proliferation-index (MIB-1) of neurofibromas in neurofibromatosis type 1 patients. Anticancer Res 23 (2A):953955, 2003 
10. George B, Lot G: Neurinomas of the first two cervical nerve roots: a series of 42 cases. J Neurosurg 82:917-923, 1995

11. Goel A, Muzumdar D, Nadkarni T, Desai K, Dange N, Chagla A: Retrospective analysis of peripheral nerve sheath tumors of the second cervical nerve root in 60 surgically treated patients. J Neurosurg Spine 8:129-134, 2008

12. Janjua MB, Zhou PL, Greenfield JP, Baaj AA, FrempongBoadu A: C2 and greater occipital nerve: the anatomic and functional implications in spinal surgery. Cureus 9:e1074, 2017

13. Jinnai T, Koyama T: Clinical characteristics of spinal nerve sheath tumors: analysis of 149 cases. Neurosurgery 56:510 515,2005

14. Leonard JR, Ferner RE, Thomas N, Gutmann DH: Cervical cord compression from plexiform neurofibromas in neurofibromatosis 1. J Neurol Neurosurg Psychiatry 78:14041406, 2007

15. Lim JK, Wong HK: Variation of the cervical spinal Torg ratio with gender and ethnicity. Spine J 4:396-401, 2004

16. Lot G, George B: Cervical neuromas with extradural components: surgical management in a series of 57 patients. Neurosurgery 41:813-822, 1997

17. Miyakoshi N, Hongo M, Kasukawa Y, Misawa A, Shimada Y: Bilateral and symmetric C1-C2 dumbbell ganglioneuromas associated with neurofibromatosis type 1 causing severe spinal cord compression. Spine J 10:e11-e15, 2010

18. Nguyen R, Dombi E, Akshintala S, Baldwin A, Widemann BC: Characterization of spinal findings in children and adults with neurofibromatosis type 1 enrolled in a natural history study using magnetic resonance imaging. J Neurooncol 121:209-215, 2015

19. Ribeiro S, Napoli I, White IJ, Parrinello S, Flanagan AM, Suter U, et al: Injury signals cooperate with Nf1 loss to relieve the tumor-suppressive environment of adult peripheral nerve. Cell Reports 5:126-136, 2013

20. Riccardi VM: The genetic predisposition to and histogenesis of neurofibromas and neurofibrosarcoma in neurofibromatosis type 1. Neurosurg Focus 22(6):E3, 2007

21. Ruggieri M, Polizzi A, Spalice A, Salpietro V, Caltabiano R, D'Orazi V, et al: The natural history of spinal neurofibromatosis: a critical review of clinical and genetic features. Clin Genet 87:401-410, 2015

22. Taleb FS, Guha A, Arnold PM, Fehlings MG, Massicotte EM: Surgical management of cervical spine manifestations of neurofibromatosis Type 1: long-term clinical and radiological follow-up in 22 cases. J Neurosurg Spine 14:356-366, 2011
23. Thakkar SD, Feigen U, Mautner VF: Spinal tumours in neurofibromatosis type 1: an MRI study of frequency, multiplicity and variety. Neuroradiology 41:625-629, 1999

24. Tsirikos AI, Saifuddin A, Noordeen MH: Spinal deformity in neurofibromatosis type-1: diagnosis and treatment. Eur Spine J 14:427-439, 2005

25. Upadhyaya M, Spurlock G, Kluwe L, Chuzhanova N, Bennett E, Thomas N, et al: The spectrum of somatic and germline NF1 mutations in NF1 patients with spinal neurofibromas. Neurogenetics 10:251-263, 2009

\section{Disclosures}

The authors report no conflict of interest concerning the materials or methods used in this study or the findings specified in this paper.

\section{Author Contributions}

Conception and design: Evans, Soh. Acquisition of data: Waqar, Huson. Analysis and interpretation of data: Waqar, Evans, Ealing. Drafting the article: Waqar. Critically revising the article: Huson, Evans, Ealing, Karabatsou, George, Soh. Reviewed submitted version of manuscript: Huson, Evans, Ealing, George, Soh. Statistical analysis: Waqar, Huson. Study supervision: Evans, Karabatsou, George, Soh.

\section{Supplemental Information \\ Previous Presentations}

This paper was presented orally at the British Neurosurgical Research Group Meeting, Birmingham, United Kingdom, March 2-3, 2017; orally at the Congress of Neurological Surgeons Annual Meeting, Boston, Massachusetts, October 7-11, 2017; and in poster form at the 17th European Neurofibromatosis Meeting, Padova, Italy, September 8-11, 2016.

\section{Correspondence}

Mueez Waqar: Salford Royal NHS Foundation Trust, Manchester, United Kingdom.mwaqar@doctors.org.uk. 